\title{
Some Orthogonal Polynomials Arising from Coherent States
}

\author{
S. Twareque Ali \\ Department of Mathematics and Statistics \\ Concordia University \\ Montréal, Québec \\ CANADA H3G $1 \mathrm{M} 8$ \\ Mourad E. H. Ismail \\ City University of Hong Kong, \\ Tat Chee Avenue, Kowloon, Hong Kong \\ and King Saud University, Riyadh, Saudi Arabia
}

April 9, 2021

\begin{abstract}
We explore in this paper some orthogonal polynomials which are naturally associated to certain families of coherent states, often referred to as nonlinear coherent states in the quantum optics literature. Some examples turn out to be known orthogonal polynomials but in many cases we encounter a general class of new orthogonal polynomials for which we establish several qualitative results.
\end{abstract}

Keywords and phrases: orthogonal polynomials, nonlinear coherent states, orthogonality measures, infinite divisibility, associated polynomials.

PACS numbers: 02.30.Sa, 03.65.Db

AMS subject classification: 46N50, 81Q99, 12 Y99

\section{Introduction}

Coherent states are well known objects, both in physics and mathematics (see, for example, [3] and references cited therein). Their use in physics goes back to the early days of quantum mechanics, starting with the 1926 paper by Schrödinger [37] and their rediscovery in the theory of optical coherence several decades later [20, 30]. In recent years coherent states have been extensively used in many areas of physics, e.g., quantum optics, atomic and molecular physics, etc., (see, for example, 22] for a detailed 
account). Their mathematical properties, specially in the context of quantization theory, square-integrable group representation theory, symplectic geometry, etc., have also been extensively studied in recent decades. In this paper we look at two sets of orthogonal polynomials that are naturally associated to a particular family of coherent states, known in the quantum optical literature as nonlinear coherent states (see [3] and [39] for an extensive discussion). One set of these polynomials, arising from the shift operators associated to these coherent states, have been known and studied before [11, 12, 34, 35]. Here we take another look at these polynomials, but study a different aspect of their structure. We also show how a second set of orthogonal polynomials can be obtained from the measure which gives the resolution of the identity for the coherent states. The moments of this measure are obtained from $n$-term partial products of the terms of the sequence defining the shift operators. In this way, both sets of orthogonal polynomials are intimately related. While it would be our aim to eventually take a closer look at the relationship between these two sets of polynomials, in the present paper we mainly focus on measures of the type which arise from the above mentioned resolution of the identity and for them discuss the associated families of orthogonal polynomials.

We start out by introducing the notion of nonlinear coherent states mathematically and the derivation of their associated polynomials, in the framework within which we would like to study them here.

\section{$1.1 \quad$ Nonlinear coherent states}

We ought to mention at the outset that the term nonlinear, as applied to coherent states, does not refer to any mathematical nonlinearity, but rather is a reflection of their appearance in nonlinear optics. Generally, a family of such coherent states is an overcomplete set of vectors in a Hilbert space, labelled by a continuous parameter $z$ which runs over a complex domain. The vectors are, in addition, subject to a resolution of the identity condition. More precisely, let $\mathfrak{H}$ be a (complex, separable, infinite dimensional) Hilbert space, $\left\{\phi_{n}\right\}_{n=0}^{\infty}$ an orthonormal basis of it and let $\left\{x_{n}\right\}_{n=0}^{\infty}, x_{0}=0$, be an infinite sequence of positive numbers. Let $\lim _{n \rightarrow \infty} x_{n}=L^{2}$, where $L>0$ could be finite or infinite, but not zero. We shall use the notation $x_{n} !=x_{1} x_{2} \ldots x_{n}$ and $x_{0} !=1$. For each $z \in \mathcal{D}$ (some domain in $\mathbb{C}$ ), we define a non-linear coherent state, i.e., a vector $\eta_{z} \in \mathfrak{H}$, in the manner

$$
\eta_{z}=\mathcal{N}\left(|z|^{2}\right)^{-\frac{1}{2}} \sum_{n=0}^{\infty} \frac{z^{n}}{\sqrt{x_{n} !}} \phi_{n}
$$

where the normalization constant $\mathcal{N}\left(|z|^{2}\right)=\sum_{n=0}^{\infty} \frac{|z|^{2 n}}{x_{n} !}$ is chosen so that $\left\|\eta_{z}\right\|=1$. It is clear that the vectors $\eta_{z}$ are well defined for all $z$ for which the above sum, representing $\mathcal{N}\left(|z|^{2}\right)$, converges, i.e., $\mathcal{D}=\{z \in \mathbb{C}|| z \mid<L\}$. Furthermore, we require that there exist a measure $d \nu(z, \bar{z})$ on $\mathcal{D}$ for which the resolution of the identity condition,

$$
\int_{\mathcal{D}}\left|\eta_{z}\right\rangle\left\langle\eta_{z}\right| \mathcal{N}\left(|z|^{2}\right) d \nu(z, \bar{z})=I_{\mathfrak{H}}
$$


holds.

It is easily seen that in order for (1.2) to be satisfied, $d \nu$ has to have the form,

$$
d \nu(z, \bar{z})=\frac{1}{2 \pi} d \theta d \lambda(r), \quad z=r e^{i \theta},
$$

where the measure $d \lambda$ is a solution of the moment problem,

$$
\int_{0}^{L} r^{2 n} d \lambda(r)=x_{n} !, \quad n=0,1,2, \ldots,
$$

provided such a solution exists. In most of the cases that occur in practice, the support of the measure $d \nu$ is the whole of $\mathcal{D}$, i.e., $d \lambda$ is supported on the entire interval $(0, L)$.

Below are some examples of the above general construction.

\subsubsection{Canonical coherent states}

Let $x_{n}=n$ so that $L=\infty$. In that case the coherent states $\eta_{z}$, defined for all $z \in \mathbb{C}$, are the well-known canonical coherent states.

$$
\eta_{z}=e^{-\frac{1}{2}|z|^{2}} \sum_{n=0}^{\infty} \frac{z^{n}}{\sqrt{n !}} \phi_{n} .
$$

The moment problem becomes,

$$
\int_{0}^{\infty} r^{2 n} d \lambda(r)=n !, \quad n=0,1,2, \ldots
$$

so that

$$
d \lambda(r)=2 r e^{-r^{2}} d r, \quad 0 \leq r<\infty
$$

\subsection{2 $\mathrm{SU}(1,1)$ discrete series coherent states}

This time $x_{n}=\frac{n}{2 j+n-1}$ so that $x_{n} !=\frac{n !}{(2 j)_{n}}$, where we have used the shifted factorials $(a)_{n}=a(a+1)(a+2) \ldots(a+n-1)$, and $j=1,1 / 2,2,3 / 2,3, \ldots$, is a constant. Also, $L=1$ and the associated coherent states, defined for all $z$ in the unit $\operatorname{disc} \mathcal{D}=|z|<1$, are

$$
\eta_{z}=\left(1-r^{2}\right)^{j} \sum_{n=0}^{\infty}\left[\frac{(2 j)_{n}}{n !}\right]^{\frac{1}{2}} z^{n} \phi_{n}, \quad r=|z| .
$$

The corresponding moment problem is now

$$
\int_{0}^{1} r^{2 n} d \lambda(r)=\frac{n !}{(2 j)_{n}}
$$

which has the solution,

$$
d \lambda(r)=2(2 j-1) r\left(1-r^{2}\right)^{2 j-2} d r, \quad 0 \leq r<1 .
$$

The coherent states, $\eta_{z}$ above, arise from the discrete series representations (parametrized by $j$ ) of the $S U(1,1)$ group. 


\subsubsection{Barut-Girardello coherent states}

There is a second set of coherent states associated to the $S U(1,1)$ group which are consructed using the ladder operators appearing in the Lie algebra of the group. These are known as the Barut-Girardello coherent states [6]. For these coherent states, $X=$ $\mathbb{C}, x_{n}=n(2 j+n-1)$ and $x_{n} !=n !(2 j)_{n}$ with $j=1,1 / 2,2,2 / 3, \ldots$, as before. The coherent states are

$$
\eta_{z}=\frac{|z|^{2 j-1}}{\sqrt{I_{2 j-1}(2|z|)}} \sum_{n=0}^{\infty} \frac{z^{n}}{\sqrt{n !(2 \kappa+n-1) !}} \phi_{n}, \quad z \in \mathbb{C}
$$

where $I_{\nu}(x)$ denotes the order- $\nu$ modified Bessel function of the first kind. These coherent states satisfy the resolution of the identity,

$$
\frac{2}{\pi} \int_{\mathbb{C}}\left|\eta_{z}\right\rangle\left\langle\eta_{z}\right| K_{2 j-1}(2 r) I_{2 j-1}(2 r) r d r d \theta=I, \quad z=r e^{i \theta},
$$

where again, $K_{\nu}(x)$ is the order- $\nu$ modified Bessel function of the second kind.

\subsubsection{Coherent states from analytic functions}

All three examples above could be seen as special cases of a more general construction. Let $f(z)$ be an analytic function which has a Taylor expansion (around the origin) of the type,

$$
f(z)=\sum_{n=0}^{\infty} \frac{z^{n}}{\rho(n)}, \quad 0<\rho(n)<\infty ;, \quad \rho(0)=1,
$$

and let $L=\lim _{n \rightarrow \infty} \frac{\rho(n+1)}{\rho(n)}>0$ be its radius of convergence. Then

$$
\mathcal{N}\left(|z|^{2}\right)=\sum_{n=0}^{\infty} \frac{|z|^{2 n}}{[\rho(n)]^{2}}
$$

converges in the disc $|z|<L$. Defining $x_{n}=\frac{\rho(n+1)}{\rho(n)}$, we can construct vectors $\eta_{z}$ in the Hilbert space $\mathfrak{H}$ following (1.1), which will be well defined in this disc and will constitute a family of coherent states provided the moment problem (1.4) has a solution. It is clear that a large number of hypergeometric functions will lead to families of coherent states in this manner.

\subsection{Associated orthogonal polynomials}

As mentioned earlier, there are two sets of orthogonal polynomials, naturally associated to a family of non-linear coherent states, that we now present. 


\subsubsection{Polynomials associated to $d \lambda$}

The first of these sets is determined by the measure $d \lambda$. This measure can be extended to an even positive measure

$$
d \mu(t)=\frac{1}{2} d \lambda(|t|),
$$

on the symmetric interval $[-L, L]$, with moments,

$$
\mu_{2 n}=2 \int_{0}^{\infty} t^{2 n} d \mu(t)=x_{n} !, \quad \mu_{2 n+1}=2 \int_{0}^{\infty} t^{2 n+1} d \mu(t)=0, n=0,1,2, \ldots .
$$

With these we can build a set of (monic) polynomials $P_{n}(x), n=0,1,2, \ldots$, orthogonal with respect to the measure $d \mu$, in the usual manner [25] using the Hankel determinant,

$$
D_{n}=\left|\begin{array}{cccc}
\mu_{0} & \mu_{1} & \ldots & \mu_{n} \\
\mu_{1} & \mu_{2} & \ldots & \mu_{n+1} \\
\vdots & \vdots & \ldots & \vdots \\
\mu_{n} & \mu_{n+1} & \ldots & \mu_{2 n}
\end{array}\right|, \quad P_{n}(x)=\frac{1}{D_{n-1}}\left|\begin{array}{cccc}
\mu_{0} & \mu_{1} & \ldots & \mu_{n} \\
\vdots & \vdots & \ldots & \vdots \\
\mu_{n-1} & \mu_{n} & \ldots & \mu_{2 n-1} \\
1 & x & \ldots & x^{n}
\end{array}\right| .
$$

For example, for the canonical coherent states with the measure (1.7), these would be Laguerre polynomials in the variable $x^{2}$. For the $S U(1,1)$ discrete series case, with the measure (1.10), the associated polynomials are related to the Jacobi polynomials as explained in Section 3.3, with $\alpha=\frac{1}{2}$ and $\beta=2 j-2$. For case of the Barut-Girardello coherent states the measure is

$$
d \lambda(r)=\frac{2}{\pi} K_{2 j-1}(2 r) r^{2-2 j} d r .
$$

The corresponding polynomials $P_{n}$ can of course be computed using (1.16), but are not among the well-known polynomials.

\subsubsection{Polynomials associated to shift operators}

To get the second set of polynomials, let us go back to the definition (1.1) and define the formal shift operators

$$
a \phi_{n}=\sqrt{x_{n}} \phi_{n-1}, \quad a \phi_{0}=0, \quad a^{*} \phi_{n}=\sqrt{x_{n+1}} \phi_{n+1}, \quad n=0,1,2, \ldots .
$$

Then, if $\sum_{n=0}^{\infty} \frac{1}{\sqrt{x_{n}}}=\infty$, the operator $Q=\frac{a+a^{*}}{\sqrt{2}}$ is essentially self-adjoint and hence has a unique self-adjoint extension [11, 35], which we again denote by $Q$. It acts on the basis vectors $\phi_{n}$ in the manner,

$$
Q \phi_{n}=\sqrt{\frac{x_{n}}{2}} \phi_{n-1}+\sqrt{\frac{x_{n+1}}{2}} \phi_{n+1} .
$$


From the general theory of self-adjoint operators, there is a Hilbert space $L^{2}(\mathbb{R}, d w)$ (where $d w$ is an even measure) on which $Q$ acts as the operator of multiplication and the $\phi_{n}$ are functions in this space. Transforming to this space we may then rewrite the above recurrence relation as:

$$
x \phi_{n}(x)=\sqrt{\frac{x_{n}}{2}} \phi_{n-1}(x)+\sqrt{\frac{x_{n+1}}{2}} \phi_{n+1}(x), \quad x \in \mathbb{R},
$$

which is a set of two-term recurrence relations for a family of orthogonal polynomials, $\phi_{n}(x)$. The polynomials can be computed successively, assuming the initial conditions, $\phi_{-1}=0, \phi_{0} \equiv 1$. The measure $d w$ comes from the spectral family of projectors, $E_{x}, x \in \mathbb{R}$, of the operator $Q$, in the manner, $d w(x)=\left\langle\phi_{0} \mid E_{x} \phi_{0}\right\rangle$.

In general, the measure $d w$ is different from $d \mu$ and so are the sets of polynomials $P_{n}(x)$ in (1.16) and $\phi(x)$ above. For example, in the case of the canonical coherent states in Section 1.1.1, the polynomials $\phi_{n}(x)$ are the well-known Hermite polynomials, while for the $S U(1,1)$ discrete series coherent states they are the Pollaczek polynomials mentioned at the end of Section [3.3, for $\beta=2 j-2$. For the Barut-Girardello CS in Section 1.1.3, $x_{n}=n(2 j+n-1)$, however again the corresponding polynomials, which can be computed using (1.20), are not well-known.

In all the above examples, the sequence $x_{n}$ is strictly increasing and its limit is either a positive number or infinity. Dickinsen, Pollack and Wannier [17] studied a class of orthogonal polynomials of the form in (1.20) but with $x_{n}$ replaced by $x_{n+\nu}$ and requiring the condition $\left\{x_{n}\right\} \rightarrow 0$. Thus, our condition (see (2.3) below) is the exact opposite of their condition. The polynomials in the Dickinsen-Pollack-Wannier class are orthogonal with respect to a discrete measure supported on a countable set whose only limit point is $x=0$. Later Goldberg [23] corrected the claim in [17] that $x=0$ does not support a mass by showing that $x=0$ may actually support a positive mass of the orthogonality measure.

There is a simple way to compute the monic versions of the polynomials $\phi_{n}(x)$. To se this, note first that in virtue of (1.19), the operator $Q$ can be represented in the $\phi_{n}$ basis as the infinite tri-diagonal matrix,

$$
Q=\left(\begin{array}{cccccc}
0 & b_{1} & 0 & 0 & 0 & \ldots \\
b_{1} & 0 & b_{2} & 0 & 0 & \ldots \\
0 & b_{2} & 0 & b_{3} & 0 & \ldots \\
0 & 0 & b_{3} & 0 & b_{4} & \ldots \\
0 & 0 & 0 & b_{4} & 0 & \ldots \\
\vdots & \vdots & \vdots & \vdots & \vdots & \ddots
\end{array}\right), \quad b_{n}=\sqrt{\frac{x_{n}}{2}}
$$

Let $Q_{n}$ be the truncated matrix consisting of the first $n$ rows and columns of $Q$ and $\mathbb{I}_{n}$ 
the $n \times n$ identity matrix. Then,

$$
x \mathbb{I}_{n}-Q_{n}=\left(\begin{array}{ccccccccc}
x & -b_{1} & 0 & 0 & 0 & \ldots & 0 & 0 & 0 \\
-b_{1} & x & -b_{2} & 0 & 0 & \ldots & 0 & 0 & 0 \\
0 & -b_{2} & x & -b_{3} & 0 & \ldots & 0 & 0 & 0 \\
0 & 0 & -b_{3} & x & -b_{4} & \ldots & 0 & 0 & 0 \\
0 & 0 & 0 & -b_{4} & x & \ldots & 0 & 0 & 0 \\
\vdots & \vdots & \vdots & \vdots & \vdots & \ddots & \vdots & \vdots & \vdots \\
0 & 0 & 0 & 0 & 0 & \ldots & x & -b_{n-2} & 0 \\
0 & 0 & 0 & 0 & 0 & \ldots & -b_{n-2} & x & -b_{n-1} \\
0 & 0 & 0 & 0 & 0 & \ldots & 0 & -b_{n-1} & x
\end{array}\right) .
$$

It now follows that the monic polynomial $q_{n}$, associated to $\phi_{n}(x)$ is just the characteristic polynomial of $Q_{n}$ :

$$
q_{n}(x)=\operatorname{det}\left[x \mathbb{I}_{n}-Q_{n}\right] .
$$

These polynomials are related to the $\phi_{n}(x)$ via

$$
q_{n}(x)=\left[\frac{x !}{2^{n}}\right]^{\frac{1}{2}} \phi_{n}(x)
$$

and satisfy the recurrence relations,

$$
q_{n+1}(x)=x q_{n}(x)-\frac{x_{n}}{2} q_{n-1}(x) .
$$

\section{Generalities about orthogonal polynomials}

To make the paper self-contained, we collect here a few preliminary results on orthogonal polynomials.

\subsection{Recurrence relations and orthogonality measures}

Every sequence of monic polynomials $\left\{P_{n}(x)\right\}$ orthogonal with respect to a positive measure satisfies a three term recurrence relation of the form [25]

$$
x P_{n}(x)=P_{n+1}(x)+\alpha_{n} P_{n}(x)+\beta_{n} P_{n-1}(x), n \geq 0, \quad P_{0}(x)=1, \beta_{0} P_{-1}(x):=0 .
$$

To avoid additional assumptions we only consider polynomials having an orthogonality measure supported on an infinite set.

We start with an even positive measure $\mu$ supported on $[-L, L], L \leq \infty$, and whose moments are $\mu_{n}, n=0,1, \cdots$. Further, normalize $\mu$ by $\mu_{0}=1$. Thus $\mu_{2 n+1}=0$ for all $n$ and $\mu_{2 n}>0$. We now assume that $\mu_{2 n}$ factors as follows

$$
\mu_{2 n}=2 \int_{0}^{L} t^{2 n} d \mu(t)=x_{1} x_{2} \ldots x_{n}=x_{n} !, \quad n>0 .
$$


(Note that this is always possible by writing $x_{n}=\mu_{2 n} / \mu_{2 n-2}$.) However, we shall only work with cases where $x_{n}$ has convenient forms, such as being expressible as rational functions of the integer variable $n$.

Note that $2 \mu$ has total mass $=1$ on $[0, L]$. The representation (2.2) clearly implies

$$
x_{n}>0, \text { for } n>0 \text {. }
$$

From here we introduce a family of orthogonal polynomials, of the type (1.20) and generated by

$$
\phi_{0}(x):=1, \quad \phi_{1}(x)=\sqrt{\frac{2}{x_{1}}} x, \quad x \phi_{n}(x)=\sqrt{\frac{x_{n+1}}{2}} \phi_{n+1}+\sqrt{\frac{x_{n}}{2}} \phi_{n-1} .
$$

We shall mainly explore in this paper the consequences of expressing the moments $\mu$ in the form (2.2) and the resulting polynomials of the above type.

\subsection{Additional background material}

It is easy to see that the quadratic form $\sum_{j, k=0}^{n} \mu_{j+k} y_{j} \overline{y_{k}}$ is positive definite, see [25] for example, hence all the Hankel determinants $D_{n}$,

$$
D_{n}:=\left|\begin{array}{cccc}
\mu_{0} & \mu_{1} & \cdots & \mu_{n} \\
\mu_{1} & \mu_{2} & \cdots & \mu_{n+1} \\
\vdots & \vdots & \cdots & \vdots \\
\mu_{n} & \mu_{n+1} & \cdots & \mu_{2 n}
\end{array}\right| .
$$

are positive, by the Sylvester criterion.

The following theorem is important in recovering the absolutely continuous component of an orthogonality measure from the large degree behavior of the orthonormal polynomials.

Theorem 2.1. Assume that $p_{n}$ satisfies (2.1) with $\alpha_{n-1} \in \mathbb{R}$ and $\beta_{n}>0$ for all $n>0$. In addition assume that

$$
\sum_{n=0}^{\infty}\left[\left|\sqrt{\beta_{n}}-\frac{1}{2}\right|+\left|\alpha_{n}\right|\right]<\infty
$$

then $\mu$ has an absolutely continuous component $\mu^{\prime}$ supported on $[-1,1]$. Furthermore if $\mu$ has a discrete part, then it will lie outside $(-1,1)$. In addition the limiting relation

$$
\limsup _{n \rightarrow \infty}\left[\sqrt{1-x^{2}} \frac{P_{n}(x)}{\sqrt{\zeta_{n}}}-\sqrt{\frac{2 \sqrt{1-x^{2}}}{\pi \mu^{\prime}(x)}} \sin ((n+1) \theta-\varphi(\theta))\right]=0
$$

holds, with $x=\cos \theta \in(-1,1)$. In (2.7) $\varphi(\theta)$ does not depend on $n$. Here $\zeta_{n}=$ $\beta_{1} \beta_{2} \cdots \beta_{n}$. 
This theorem is due to Nevai [33]. Observe that Nevai's theorem then relates the asymptotics of general polynomials to those of the Chebyshev polynomials. Note also that Nevai's theorem gives a scattering amplitude and says that there may be a phase shift.

Theorem 2.2. Let $p_{n}(x)$ be generated by (2.1). Then the zeros of the polynomial $p_{n}(x)$ lie in $(A, B)$, where

$$
B=\max \left\{\xi_{j}: 0<j<n\right\}, \quad A=\min \left\{\eta_{j}: 0<j<n\right\},
$$

where $\eta_{j} \leq \xi_{j}$ and

$$
\xi_{j}, \eta_{j}=\frac{1}{2}\left(\alpha_{j}+\alpha_{j-1}\right) \pm \frac{1}{2} \sqrt{\left(\alpha_{j}-\alpha_{j-1}\right)^{2}+16 \beta_{j}}, \quad 1 \leq j<n .
$$

Theorem 2.2 is the special case $c_{n}=1 / 4$ of a result due to Ismail and Li in [27]. The full result is also stated and proved in [25, Theorem 7.2.7].

The zeros of orthogonal polynomials are real and simple, so we shall follow the standard notation in [25] or 38] and arrange the zeros $x_{n, j}, 1 \leq j \leq n$ as

$$
x_{n, 1}>x_{n, 2}>\cdots>x_{n, n} .
$$

Berg and Duran [9] proved the following theorem.

Theorem 2.3. Assume that $\left\{1 / x_{n}\right\}$ is a Hausdorf moment sequence, that is there is a probability measure $\nu$ supported on $[0,1]$ such that $1 / x_{n}=\int_{0}^{1} t^{n} d \nu(x)$. Then there is a probability measure $\xi$ upported on $[0, \infty)$ such that $x_{1} x_{2} \cdots x_{n}=\int_{0}^{\infty} t^{n} d \xi(x)$, that is $\left\{x_{1} x_{2} \cdots x_{n}\right\}$ is a Stieltjes moment sequence.

Many examples and applications are in [7], 8]. In the theory of coherent states we start with the sequence $\left\{x_{n}\right\}$ and construct the measure $\lambda$, so Theorem 2.3 gives a sufficient condition for the existence of the measure $\lambda$.

\section{$3 \quad$ Results and examples}

We enunciate a few results in this section and work out some examples.

\subsection{Bounds}

Our first result is the monotonicity of $\left\{x_{n}\right\}$.

Theorem 3.1. The sequence $\left\{x_{n}: n=1,2, \cdots\right\}$ is strictly increasing. If $L<\infty$ then $x_{n}<L^{2}$.

Proof. Clearly

$$
0<D_{2}=\left|\begin{array}{ccc}
1 & 0 & x_{1} \\
0 & x_{1} & 0 \\
x_{1} & 0 & x_{1} x_{2}
\end{array}\right|=x_{1}^{2}\left(x_{2}-x_{1}\right)
$$


Therefore $x_{2}>x_{1}$. We note that $x^{2 n} d \mu$ is a positive even measure supported on $[-L, L]$ and its $D_{2}$ is

$$
\left|\begin{array}{ccc}
\mu_{2 n} & 0 & \mu_{2 n+2} \\
0 & \mu_{2 n+2} & 0 \\
\mu_{2 n+2} & 0 & \mu_{2 n+4}
\end{array}\right|=\mu_{2 n+2}\left[\mu_{2 n}\right]^{2}\left[x_{n+1} x_{n+2}-x_{n+1}^{2}\right]
$$

which implies $x_{n+2}>x_{n+1}$, for $n=0,1, \cdots$. The same conclusion also follows from

$$
\begin{gathered}
0<2 \int_{0}^{L} t^{2 n}\left(t^{2}-x_{n+1}\right)^{2} d \mu(t)=\mu_{2 n+4}-2 x_{n+1} \mu_{2 n+2}+x_{n+1}^{2} \mu_{2 n} \\
=\mu_{2 n+2}\left[x_{n+2}-2 x_{n+1}+x_{n+1}\right] .
\end{gathered}
$$

If $L$ is finite we use the definition of $\mu_{2 n}=x_{1} \cdots x_{n}$ and conclude that

$$
x_{1} \cdots x_{n} L^{2}-x_{1} \cdots x_{n} x_{n+1}=2 \int_{0}^{L} t^{2 n}\left[L^{2}-t^{2}\right] d \mu(x)>0 .
$$

This implies $x_{n+1}<L^{2}$ for all $n \geq 0$.

A partial converse to the above theorem is the following.

Theorem 3.2. If $L=\infty$ then the sequence $\left\{x_{n}\right\}$ is unbounded.

Proof. Assume $L=\infty$ and $x_{n} \leq M$, and $M>1$. Then for every $A>0$ the integral $\int_{[A, \infty)} d \mu(t)>0$. It is clear that

$$
\begin{aligned}
& M^{n}>x_{1} x_{2} \cdots x_{n}=2 \int_{[0, \infty)} t^{2 n} d \mu(t) \geq 2 \int_{[2 M, \infty)} t^{2 n} d \mu(t) \\
& \geq 2[2 M]^{2 n} \int_{[M, \infty)} d \mu(t)
\end{aligned}
$$

Therefore $M>4 M^{2}\left[2 \int_{[2 M, \infty)} d \mu(t)\right]^{1 / n}$ which is impossible for sufficiently large $n$.

Note that if $L \leq 1$ then $x_{n}<1$ and $1 / x_{n}$ is never a Hausdorff moment sequence. In these cases the assumptions of the Berg-Durand theorem, Theorem 2.3, are not satisfied.

Remark: It is not clear that we can say much about the case $L=\infty$ so we will assume that $L$ is bounded throughout the rest of this section. Therefore $\left\{x_{n}\right\}$ is a monotone sequence converging to $M$, say. One can derive nonlinear inequalities satisfied by the $x_{n}$ 's. For example using

$$
0<2 \int_{0}^{L} t^{2 n}\left(t^{2}-x_{n+1}\right)^{2}\left(t^{2}-x_{n+2}\right)^{2} d \mu(t)
$$

one gets

$$
\begin{gathered}
x_{n+3}\left(x_{n+4}-x_{n+2}\right)+x_{n+1}\left(x_{n+2}-x_{n+1}\right) \\
>x_{n+2}\left(x_{n+3}-x_{n+2}\right)+2 x_{n+1}\left(x_{n+3}-x_{n+2}\right) .
\end{gathered}
$$


Of course one can integrate other factions like $t^{2 n}\left(t^{2}-x_{n+1}\right)^{4}$ or $t^{2 n}\left(t^{2}-x_{n+1}\right)^{2}\left(t^{2}-x_{n+3}\right)^{2}$ and get other inequalities. On the other hand by expanding the determinant Hankel determinant

$$
\left|\begin{array}{ccccc}
\mu_{2 n} & 0 & \mu_{2 n+2} & 0 & \mu_{2 n+4} \\
0 & \mu_{2 n+2} & 0 & \mu_{2 n+4} & 0 \\
\mu_{2 n+2} & 0 & \mu_{2 n+4} & 0 & \mu_{2 n+6} \\
0 & \mu_{2 n+4} & 0 & \mu_{2 n+6} & 0 \\
\mu_{2 n+4} & 0 & \mu_{2 n+6} & 0 & \mu_{2 n+8}
\end{array}\right|>0
$$

and after deleting the positive terms we obtain the necessary condition

$$
2 x_{n+1} x_{n+2} x_{n+3}+x_{n+2} x_{n+3} x_{n+4}>x_{n+1} x_{n+1}^{2}+x_{n+2} x_{n+3}^{2}+x_{n+1} x_{n+3} x_{n+4} .
$$

Theorem 3.3. Let $L$ be finite and let $M:=\lim _{n \rightarrow \infty} x_{n}$. If

$$
\sum_{n=1}^{\infty}\left|\sqrt{x_{n}}-\sqrt{M}\right| \text { converges. }
$$

Then the orthogonality measure of $\phi_{n}(x)$ has an absolutely continuous component supported on the interval $[-2 \sqrt{M}, 2 \sqrt{M}]$. Moreover all the zeros of the polynomials lie in $(-2 \sqrt{M}, 2 \sqrt{M})$, hence the discrete part of the orthogonality measure is either empty or has two discrete masses (bound states) at $x= \pm 2 \sqrt{M}$.

Proof. Let $x=y / 2 \sqrt{M}$ and put $\phi_{n}(x)=\psi_{n}(y)$ and apply Theorem 2.1 to see that $\psi_{n}$ 's are orthogonal with respect to a measure whose absolutely continuous component is supported on $[-1,1]$. Thus the orthogonality measure of $\phi_{n}$ has an absolutely continuous component supported on $[-2 \sqrt{M}, 2 \sqrt{M}]$. Next apply Theorem 2.2. In the present case $\alpha_{n}=0, \beta_{n}=x_{n}$, hence $\xi_{j}, \eta_{j}= \pm 2 \sqrt{x_{j}}$. The monotonicity of the $x_{n}$ s shows that the zeros of $\phi_{n}(x)$ belong to $\left(-\sqrt{x_{n}}, \sqrt{x_{n}}\right)$. From Theorem 2.1 we conclude that the discrete part of the orthogonality measure is outside $(-2 \sqrt{M}, 2 \sqrt{M})$. If $[A, B]$ is the smallest interval containing the support of the orthogonality measure then the largest and smallest zeros of $\phi_{n}$ converge to $B$ and $A$, respectively. Thus $[A, B]=[-2 \sqrt{M}, 2 \sqrt{M}]$. This shows that the discrete part may only occur at $\pm 2 \sqrt{M}$.

\subsection{Example 1}

Consider the ultraspherical polynomials $\left\{C_{n}^{\nu}(x)\right\}$ where

$$
d \mu(x)=\frac{\Gamma(\nu+1)\left(1-x^{2}\right)^{\nu-1 / 2}}{\sqrt{\pi} \Gamma(\nu+1 / 2)} d x, \quad x \in[-1,1], \nu>-1 / 2 .
$$

Now

$$
\begin{gathered}
x_{1} x_{2} \cdots x_{n}=\frac{2 \Gamma(\nu+1)}{\sqrt{\pi} \Gamma(\nu+1 / 2)} \int_{0}^{1} x^{2 n}\left(1-x^{2}\right)^{\nu-1 / 2} d x \\
=\frac{\Gamma(\nu+1) \Gamma(n+1 / 2)}{\sqrt{\pi} \Gamma(\nu+n+1)}=\frac{(1 / 2)_{n}}{(\nu+1)_{n}}
\end{gathered}
$$


Therefore

$$
x_{n}=\frac{n-1 / 2}{\nu+n} .
$$

Here is an interesting point to show how sharp the monotonicity of the $x_{n}$ 's is. An easy calculation is to show that $x_{n}<x_{n+1}$ is equivalent to the integrability of the weight function, namely $\nu>-1 / 2$. The monic recurrence relation for the family of polynomials in (2.4), after replacing $x$ by $2 x$, is

$$
2 x u_{n}(x)=u_{n+1}(x)+\frac{n-1 / 2}{\nu+n} u_{n-1}(x),
$$

which is not a standard polynomial. It is a special case of the associated Pollaczek polynomials, see [25, Chapter 5]. We know the absolutely continuous component of its orthogonality measure but we do not know whether $x= \pm 1$ support any discrete masses.

\subsection{Example 2}

This is more general than Example 1. Consider the absolutely continuous measure

$$
d \mu(x ; \alpha, \beta)=\frac{\Gamma(\alpha+\beta+3 / 2)|x|^{2 \alpha}\left(1-x^{2}\right)^{\beta}}{\Gamma(\alpha+1 / 2) \Gamma(\beta+1)} d x, \quad x \in[-1,1],
$$

where $\alpha>-1 / 2, \beta>-1$. This is essentially the measure $\lambda$ in (1.4). The polynomials in this case are defined according to their parity. The polynomials of even degree are constant multiples of the Jacobi polynomials $P_{n}^{(\alpha-1 / 2, \beta)}\left(1-2 x^{2}\right)$ while the odd degree ones are constant multiples of the Jacobi polynomials $x P_{n}^{(\alpha+1 / 2, \beta)}\left(1-2 x^{2}\right)$.

We then have

$$
\begin{gathered}
x_{1} x_{2} \cdots x_{n}=\frac{\Gamma(\alpha+\beta+3 / 2)}{\Gamma(\alpha+1 / 2) \Gamma(\beta+1)} \int_{0}^{1} x^{2 n+2 \alpha}\left(1-x^{2}\right)^{\beta} d x \\
=\frac{(\alpha+1 / 2)_{n}}{(\alpha+\beta+3 / 2)_{n}} .
\end{gathered}
$$

This gives

$$
x_{n}=\frac{\alpha+n-1 / 2}{\alpha+\beta+n+1 / 2} .
$$

The case $\alpha=1 / 2$ gives the Pollaczek polynomials with parameters $\lambda=(\beta+1) / 2, a=$ $(\beta+1) / 2, b=0$, see $\S 5.4$ and $\S 5.5$ of [25]. If $\alpha \neq 1 / 2$ we get the associated Pollaczek polynomials. They are given at the end of [15].

For completeness It may be of interest to say some thing about the Pollaczek polynomials. They can be defined by the recurrence relation, [16], [25]

$$
\begin{gathered}
(n+1) P_{n+1}^{\lambda}(x ; a, b)=2[(n+\lambda+a) x+b] P_{n}^{\lambda}(x ; a, b) \\
-(n+2 \lambda-1) P_{n-1}^{\lambda}(x ; a, b), \quad n>0,
\end{gathered}
$$

and the initial conditions

$$
P_{0}^{\lambda}(x ; a, b)=1, \quad P_{1}^{\lambda}(x ; a, b)=2(\lambda+a) x+2 b .
$$


Their hypergeometric representation, orthogonality relation and generating functions can be found in $\S 5.3$ of 25 . The orthogonality restricts $\lambda . a, b$ to be in a certain subset of $\mathbb{R}^{3}$. The parameter domain is further divided into subsets according to the nature of their orthogonality measure. The measure always has an absolutely continuous component supported on $[-1,1]$. In addition it may have an empty, finite or infinite discrete part depending on where $\lambda, a, b$ lies in the parameter domain. This is described in detail in [15], see also $§ 5.3$ in [25]. The Pollaczek polynomials also appeared in the $J$ matrix method for discretization of the continuum where the energy parameter $E$ for the hydrogen atom is related to $x$ in the Pollaczek polynomials via $x=(E-1 / 8) /(E+1 / 8$. The details are in [41], [26], see also [25, §5.8]. The latter reference records the explicit form of the measure in different parts of the parameter domain. It is interesting to note that the Pollaczek polynomials also appear in the relativistic Coulomb problem as in the work of Abdulaziz Alhaidari [2] and Charles Munger 32].

\subsection{Further examples}

We consider two additional examples of measures, with moments written in the form (2.2). Consider the integral, [18, (27),p.51],

$$
\left.\int_{0}^{\infty} K_{2 \nu}(\beta t) t^{2 \mu-1} d t=2^{2 \mu-2} \beta^{-2 \mu} \Gamma(\mu+\nu) \Gamma(\mu-\nu)\right),
$$

$\Re(\mu \pm \nu+>0, \Re \beta>0$. The weight function

$$
w(x)=\frac{2^{1-2 \mu} \beta^{2 \mu}}{\Gamma(\mu+\nu) \Gamma(\mu-\nu))} K_{2 \nu}(\beta|x|)|x|^{2 \mu-1}, \quad x \in \mathbb{R} .
$$

Therefore

$$
\mu_{2 n}=4^{n} \beta^{-2 n}(\mu+\nu)_{n}(\mu-\nu)_{n}
$$

and

$$
x_{n}=\left(4 / \beta^{2}\right)(\mu+\nu+n-1)(\mu-\nu+n-1) .
$$

In this case the polynomials generated by (2.4) are the associated Meixner-Pollaczek polynomials. Weight functions for these polynomials have been computed in [15].

Consider the integral

$$
\begin{gathered}
\int_{0}^{\infty} e^{-a t} K_{\nu}(\beta t) t^{\mu-1} d t=\frac{\sqrt{\pi}(2 \beta)^{\nu} \Gamma(\mu+\nu) \Gamma(\mu-\nu)}{\Gamma(\mu+1 / 2)(a+\beta)^{\mu+\nu}} \\
\times_{2} F_{1}\left(\begin{array}{c|c}
\mu+\nu, \nu+1 / 2 & \frac{a-\beta}{a+\beta} \\
\mu+1 / 2 &
\end{array}\right.
\end{gathered}
$$

valid for $\Re(\mu \pm \nu>0, \Re(a+\beta)>0$. This is $(26)$, page 50 of [18]. 
Eample 1: To sum the ${ }_{2} F_{1}$ we are forced to take $a=\beta$, hence there is no loss of generality in choosing $a=\beta=1$. Consider the even normalized weight function

$$
w(t):=\frac{\Gamma(\mu+1 / 2) 2^{\mu}}{\sqrt{\pi} \Gamma(\mu+\nu) \Gamma(\mu-\nu)} e^{-t^{2}} K_{\nu}\left(t^{2}\right)|t|^{2 \mu-1}, \quad t \in \mathbb{R} .
$$

Thus

$$
\mu_{2 n}=\frac{(\mu+\nu)_{n}(\mu-\nu)_{n}}{2^{n}(\mu+1 / 2)_{n}}
$$

and we find $x_{n}$ in (2.2) is

$$
x_{n}=\frac{(\mu+\nu+n-1)(\mu-\nu+n-1)}{2(\mu+n-1 / 2)} .
$$

The $x_{n}$ s are unbounded as expected. Nothing is known about the polynomials generated by (2.4) with $x_{n}$ defined by (3.14).

Let

$$
w(t):=\frac{\Gamma(\mu+1 / 2) 2^{\mu-1}}{\sqrt{\pi} \Gamma(\mu+\nu) \Gamma(\mu-\nu)} e^{-|t|} K_{\nu}(|t|)|t|^{\mu-1}, \quad t \in \mathbb{R} .
$$

Thus

$$
\mu_{2 n}=\frac{(\mu+\nu)_{2 n}(\mu-\nu)_{2 n}}{4^{n}(\mu+1 / 2)_{2 n}}
$$

and we find for $x_{n}$ in (2.2)

$$
x_{n}=\frac{(\mu+\nu+2 n-2)(\mu+\nu+2 n-1)(\mu-\nu+2 n-2)(\mu-\nu+2 n-1)}{4(\mu+2 n-3 / 2)(\mu+2 n-1 / 2)} .
$$

At first glance these polynomials seem to be symmetric continuous Hahn polynomials, [31, (1.4.2)] with $a=c$ and $b=d$. A closer examination however shows that this is not the case and the polynomials generated by (2.4) with the above $x_{n}$ 's are new.

\subsection{Completely Monotonic Functions}

Bustoz and Ismail [14] proved that the function

$$
f(x ; a, b):=\frac{\Gamma(x) \Gamma(x+a+b)}{\Gamma(x+a) \Gamma(x+b)}, \quad a, b \geq 0,
$$

is completely monotonic, that is $(-1)^{n} \frac{d^{n}}{d x^{n}} f(x, a, b) \geq 0$ on $(0, \infty)$. Therefore the function

$$
g(x ; a, c):=\frac{\Gamma(a) \Gamma(b)}{\Gamma(c) \Gamma(a+b-c)} \frac{\Gamma(x+c) \Gamma(x+a+b-c)}{\Gamma(x+a) \Gamma(x+b)},
$$


is completely monotonic for $a \geq c, b \geq c, c \geq 0$. When $c>0, g$ is completely monotonic on $[0, \infty)$. By Bernstein's theorem [40] there is a unique probability measure $\alpha(x)$ supported on a subset of $[0, \infty)$ such that

$$
g(x ; a, b, c)=\int_{0}^{\infty} e^{-x t} d \alpha(t)
$$

In fact Bustoz and Ismail [13] proved that the corresponding probability distribution is infinitely divisible [19]. Now the measure $\mu(u):=\frac{1}{2} \alpha(-2 \ln |u|)$ is an even probability measure on $\mathbb{R}$ and its $2 n$-th moment is

$$
\int_{\mathbb{R}} u^{2 n} d \mu(u)=2 \int_{0}^{1} u^{2 n} d \mu(u)=g(n ; a, b, c)=x_{1} x_{2} \cdots x_{n} .
$$

This gives

$$
x_{n}=\frac{(c+n-1)(a+b-c+n-1)}{(a+n-1)(b+n-1)} .
$$

The polynomials generated by (2.4) when $c=1, a=\nu+1, b=\nu$ are the orthonormal ultraspherical polynomials

$$
\sqrt{\frac{n !(n+\nu)}{(2 \nu)_{n}}} C_{n}^{\nu}(x / 2)
$$

[36], 18], 25. In general we choose $a=\nu+1+c, b=\nu+c$ and keep $c$ as an association parameter. The polynomials become constant multiples of orthonormal associated ultraspherical polynomials at $x / 2,[13]$.

Let $0<q<1$. The $q$-shifted factorials are

$$
(a ; q)_{0}=1,(a ; q)_{n}=\prod_{k=1}^{n}\left(1-a q^{k-1}\right), \quad n=1,2, \cdots, \text { or } \infty .
$$

The $q$-Gamma function $\Gamma_{q}(x)$ is, [4, 21]

$$
\Gamma_{q}(x)=(1-q)^{1-x} \prod_{k=0}^{\infty} \frac{1-q^{k+1}}{1-q^{x+k}} .
$$

It satisfies the functional equation

$$
\Gamma_{q}(x+1)=\frac{1-q^{x}}{1-q} \Gamma_{q}(x)
$$

It also has the initial values $\Gamma_{q}(1)=\Gamma_{q}(2)=1$.

We now consider the function

$$
h(x ; a, c):=\frac{\Gamma_{q}(a) \Gamma_{q}(b)}{\Gamma_{q}(c) \Gamma_{q}(a+b-c)} \frac{\Gamma_{q}(x+c) \Gamma_{q}(x+a+b-c)}{\Gamma_{q}(x+a) \Gamma(x+b)} .
$$


A special case of a result of Ismail and Muldoon [29] is that $h(x ; a, c)=e^{-H(t)}$ and $H$ is completely monotonic on $[0, \infty)$ for $a \geq c, b \geq c, c \geq 0$.

Now let $\beta$ be the probability measure defined by

$$
h(x ; a, c)=\int_{0}^{\infty} e^{-x t} d \beta(t) .
$$

As in the case of (3.19) we let $\nu(u):=\frac{1}{2} \beta(-2 \ln |u|)$ and set

$$
\int_{\mathbb{R}} u^{2 n} d \mu(u)=2 \int_{0}^{\infty} u^{2 n} d \mu(u)=g(n ; a, b, c)=x_{1} x_{2} \cdots x_{n} .
$$

With $A=q^{a}, B=q^{b}, C=q^{c}, D=q^{d}$, we have

$$
x_{n}=\frac{\left(1-C q^{n-1}\right)\left(1-A B C q^{n-1}\right)}{\left(1-A q^{n-1}\right)\left(1-B q^{n-1}\right)} .
$$

When $C=q, A / q=B=\beta$ the polynomials are the $q$-ultraspherical polynomials of Askey and Ismail [5]

$$
\sqrt{\frac{(q ; q)_{n}\left(1-\beta q^{n}\right)}{\left(\beta^{2} ; q\right)_{n}}} C_{n}(x / 2 ; \beta \mid q) .
$$

A complete treatment of the $q$-ultraspherical polynomials is available in [25]. If $C \neq q$ we get the associated $q$-ultraspherical polynomials of [13] or associated symmetric $q$ Pollaczek polynomials, [15.

Many quotients of products of Gamma (repectively q-Gamma) functions are known to be completely monotonic, see for example [24] and [29]. Each combination gives rise to orthogonal polynomials $\phi_{n}(x)$ of the type generated by (2.4), where $x_{n}$ is a quotient of two monic polynomials of $n$ (respectively of $q^{n}$ ) of the same degree. Therefore we can always generate many cases which are not in the literature but to which the results of this section apply. We end this section with few examples of completely monotonic functions and the write down the corresponding sequence $\left\{x_{n}\right\}$.

In order to state the more general results alluded to above we need some additional notation. Let $S_{n}$ be the set (group) of all permutations on $n$ symbols, $a_{1}, a_{2}, \ldots, a_{n}$. Let $O_{n}$, and $E_{n}$ be the sets of odd, and even permutations over $n$ symbols, respectively. Moreover let $P_{n, k} ; 1 \leq k \leq n$ be the set of all vectors $\mathbf{m}=\left(m_{1}, m_{2}, \cdots, m_{k}\right)$ such that $1 \leq m_{r}<m_{s} \leq n$ for $1 \leq r<s \leq k$; and $P_{n, 0}$ is defined as the empty set.

Theorem 3.4. ([24]) Let $a_{1}>a_{2}>\cdots>a_{n} \geq 0$ and define

$$
F(x)=\frac{\prod_{\sigma \in E_{n}}\left[\Gamma\left(x+a_{\sigma(2)}+2 a_{\sigma(3)}+\cdots+(n-1) a_{\sigma(n)}\right]\right.}{\prod_{\sigma \in O_{n}}\left[\Gamma\left(x+a_{\sigma(2)}+2 a_{\sigma(3)}+\cdots+(n-1) a_{\sigma(n)}\right)\right]} .
$$

Then $F\left(x-a_{2}-2 a_{3}-\cdots-(n-1) a_{n}\right)=e^{-H(x)}$ and $H^{\prime}$ is completely monotonic, hence $F$ is completely monotonic. The same conclusion holds for the function

$$
F(x, q)=\frac{\prod_{\sigma \in E_{n}}\left[\Gamma_{q}\left(x+a_{\sigma(2)}+2 a_{\sigma(3)}+\cdots+(n-1) a_{\sigma(n)}\right]\right.}{\prod_{\sigma \in O_{n}}\left[\Gamma_{q}\left(x+a_{\sigma(2)}+2 a_{\sigma(3)}+\cdots+(n-1) a_{\sigma(n)}\right)\right]} .
$$


Note that

$$
\sum_{k=1}^{n}(k-1)\left(a_{\sigma(k)}-a_{k}\right) \geq 0
$$

holds for any permutation $\sigma$ when $a_{1}>a_{2}>\cdots>a_{n}>0$.

Theorem 3.5. The function

$$
F_{s}(x)=\frac{\Gamma(x) \prod_{k=1}^{\lfloor s / 2\rfloor}\left[\prod_{\mathbf{m} \in P_{s, 2 k}} \Gamma\left(x+\sum_{j=1}^{2 k} a_{m_{j}}\right]\right.}{\prod_{k=1}^{\lfloor(s+1) / 2\rfloor}\left[\prod_{\mathbf{m} \in P_{s, 2 k-1}} \Gamma\left(x+\sum_{j=1}^{2 k-1} a_{m_{j}}\right]\right.},
$$

is of the form $e^{-H(x)}$ and $H^{\prime}(x)$ is completely monotonic, hence $F_{n}$ is completely monotonic.

Theorem 3.5 is due to Grinshpan and Ismail [24]. In particular the case $n=3$, after shifting $x$ by $a_{0}$, says that the function

$$
\frac{\Gamma\left(x+a_{0}\right) \Gamma\left(x+a_{0}+a_{1}+a_{2}\right) \Gamma\left(x+a_{0}+a_{1}+a_{3}\right) \Gamma\left(x+a_{0}+a_{2}+a_{3}\right)}{\Gamma\left(x+a_{0}+a_{1}\right) \Gamma\left(x+a_{0}+a_{2}\right) \Gamma\left(x+a_{0}+a_{3}\right) \Gamma\left(x+a_{0}+a_{1}+a_{2}+a_{3}\right)}
$$

is completely monotonic for $a_{1} \geq a_{2} \geq a_{3} \geq 0$, and $a_{0} \geq 0$. Choosing $a_{0}=1$ and dividing the above function by its value at $x=a_{0}=1$ we find that the corresponding $x_{n}$ 's are given by

$$
x_{n}=\frac{n\left(n+a_{1}+a_{2}\right)\left(n+a_{1}+a_{3}\right)\left(n+a_{2}+a_{3}\right)}{\left(n+a_{1}\right)\left(n+a_{2}\right)\left(n+a_{3}\right)\left(n+a_{1}+a_{2}+a_{3}\right)} .
$$

In this case $x_{n} \rightarrow 1$ and $\left|\sqrt{x_{n}}-1\right|=\mathcal{O}\left(1 / n^{2}\right)$, as $n \rightarrow \infty$, hence $\sum_{n=1}^{\infty}\left|\sqrt{x_{n}}-1\right|<\infty$ and the conclusions of Nevai's theorem hold.

Remark 3.6. Let $\mu$ be a probability measure such that

$$
F_{s}\left(x+a_{0}\right) / F_{s}\left(a_{0}\right)=\int_{0}^{\infty} e^{-x t} d \mu(t)=\int_{0}^{1} u^{2 x} d \nu(u)
$$

where we performed the change of variables $e^{-t / 2}=u$. Therefore

$$
F_{s}\left(n+a_{0}\right) / F_{s}\left(a_{0}\right)=\int_{0}^{1} u^{2 n} d \nu(u)=\int_{-1}^{1} u^{2 n}\left[\frac{1}{2} d \nu(|u|)\right],
$$

where $\nu$ is extended as an even measure, so that $\frac{1}{2} d \nu$ is now a probability measure on $[-1,1]$. It is clear we can define $x_{n}$ by

$$
x_{n}=F_{s}\left(n+a_{0}\right) / F_{s}\left(n+a_{0}-1\right), n \geq 1 .
$$

We will show below that the $x_{n}$ s defined this way have the property $\left|\sqrt{x_{n}}-1\right|=\mathcal{O}\left(1 / n^{2}\right)$, $n \rightarrow \infty$, so Nevai's theorem is applicable. 
We now show that the $x_{n}$ 's defined by (3.29) satisfy the conditions in Nevai's theorem. Observe that the number of terms in the numerator in (3.26) is

$$
1+\left(\begin{array}{l}
s \\
2
\end{array}\right)+\left(\begin{array}{l}
s \\
4
\end{array}\right)+\cdots+\left(\begin{array}{c}
s \\
2\lfloor s / 2\rfloor
\end{array}\right)=2^{s-1},
$$

while the number of terms in the denominator in (3.26) is

$$
\left(\begin{array}{l}
s \\
1
\end{array}\right)+\left(\begin{array}{l}
s \\
3
\end{array}\right)+\left(\begin{array}{l}
s \\
5
\end{array}\right)+\cdots+\left(\begin{array}{c}
s \\
2\lfloor(s+1) / 2\rfloor
\end{array}\right)=2^{s-1} .
$$

So the number of terms in the numerator and denominator in (3.26) is the same. Now the sum of the arguments of the Gamma functions in the numerator and denominator in (3.26) is

$$
\begin{gathered}
2^{s-1}+2\left(\begin{array}{l}
s \\
2
\end{array}\right)+4\left(\begin{array}{l}
s \\
4
\end{array}\right)+\cdots+2\lfloor s / 2\rfloor\left(\begin{array}{c}
s \\
2\lfloor s / 2\rfloor
\end{array}\right), \quad \text { and } \\
2^{s-1}+\left(\begin{array}{l}
s \\
1
\end{array}\right)+3\left(\begin{array}{l}
s \\
3
\end{array}\right)+5\left(\begin{array}{l}
s \\
5
\end{array}\right)+\cdots+2\lfloor(s+1) / 2\rfloor\left(\begin{array}{c}
s \\
2\lfloor(s+1) / 2\rfloor
\end{array}\right) .
\end{gathered}
$$

respectively. But both are equal to $2^{s-1}+s 2^{s-2}$. After discarding the $x^{\prime} s$ each sum of the remaining terms is $s 2^{s-2}$. But both the numerator and denominator sums are symmetric functions of the $a_{j}$ s hence each $a_{j}$ appears $2^{s-2}$ times. This and the monotonicity of $F_{s}$ show that $1>x_{n}=1-\mathcal{O}\left(1 / n^{2}\right)$.

It would be of great interest to study the coherent states arising from the above weight functions and associated sequences $\left\{x_{n}\right\}$. Many of these are expected to have physical significance.

\section{Acknowledgements}

The research of Mourad E.H. Ismail is supported by NPST Program of King Saud University; project number 10-MAT1293-02 and by Research Grants Council of Hong Kong under contract \# 101410. The work of S.T. Ali was partially supported by a grant from the Natural Science and Engineering Research Council (NSERC) of Canada. Also part of this work was carried out while STA was visiting MEHI at the City University of Hong Kong. He would like to thank the Department of Mathematics, City University of Hong Kong, for hospitality.

\section{References}

[1] N. I. Akhiezer, The Classical Moment Problem and Some Related Questions in Analysis, English translation, Oliver and Boyed, Edinburgh,1965.

[2] A.D. Al-Haidari, Exact $L^{2}$-series solution of the Dirac-Coulomb problem for all energies, Ann. Physics 312 (2004) 144-160. 
[3] S.T. Ali, J.-P. Antoine and J.P. Gazeau, Coherent States, Wavelets and their Generalizations, Springer, New York, 2000.

[4] G. E. Andrews, R. A. Askey, and R. Roy, Special Functions, Cambridge University Press, Cambridge, 1999.

[5] R. Askey and M. E. H. Ismail, A generalization of the ultraspherical polynomials, in "Studies in Pure Mathematics", edited by P. Erdős, Birkhauser Verlag, Basel, $1983,55-78$.

[6] A.O.Barut and L. Girardello, New "coherent" states associated with non-compact groups, Commun. Math. Phys. 21 (1971), 41-55.

[7] C. Berg, On powers of Stieltjes moment sequences I, J. Theoretical Probability 18 (2005), 871-889

[8] C. Berg, On powers of Stieltjes moment sequences I, II, J. Comp. Appl. Math. 199 (2007), 23-38.

[9] C. Berg and A. J. Duran, A transformation from Hausdorff to Stieltjes moment problem sequences, Ark. Mat 42 (2004), 239-257.

[10] Y. M. Berezansky, Expansions in Eigenfunctions od Selfadjoint Operators, English translation, American Mathematical Society, Providence, Rhode Island, 1968.

[11] V.V. Borzov, Orthogonal polynomials and generalized oscillator algebras, Integral Transforms Spec. Funct. 12 (2001), 115-138.

[12] V.V.Borzov and E.V.Damaskinsky, Realization of the annihilation operator for an oscillator-like system by a differential operator and Hermite-Chihara polynomials, Integral Transforms Spec. Funct. 13 (2002), 18.

[13] J. Bustoz and M. E. H. Ismail, The associated classical orthogonal polynomials and their q-analogues, Canadian J. Math. 34 (1982), 718-736.

[14] J. Bustoz and M. E. H. Ismail, On gamma function inequalities, Mathematics of Computation 47 (1986), 659-667

[15] J. Charris and M. E. H. Ismail, On sieved orthogonal polynomials V: Sieved Pollaczek polynomials, SIAM J. Math. Anal. 18 (1987), 1177-1218.

[16] T. S. Chihara, An Introduction to Orthogonal Polynomials, Gordon and Breach, New York, 1978, reprinted by Dover, New York, 2009.

[17] D.J. Dickinson and H.O. Pollack and G.H. Wannier, On a class of polynomials orthogonal over a denumerable set, Pacific J. Math, 6 (1956), 239-247.

[18] A. Erdélyi, W. Magnus, F. Oberhettinger, and F. G. Tricomi, Higher Transcendental Functions, volume 2, McGraw-Hill, New York, 1953. 
[19] W. Feller, An Introduction to Probability Theory and Its Applications, vol. 2, Academic Press, New York, 1966.

[20] R. J. Glauber, The quantum theory of optical coherence, Phys. Rev. 130 (1963) 25292539; and Coherent and incoherent states of radiation field, ibid. 131 (1963) 27662788.

[21] G. Gasper and M. Rahman, Basic Hypergeometric Series, second edition Cambridge University Press, Cambridge, 2004.

[22] J.-P. Gazeau, Coherent States in Quantum Physics, Wiley-VCH, Weinheim, 2009.

[23] J. Goldberg, Polynomials orthogonal over a denumerable set, Pacific J. Math., 15 (1965), 1171-1186.

[24] A. Z. Grinshpan and M. E. H. Ismail, Completely monotonic functions involving the Gamma and q-Gamma functions, Proc. Amer. Math. Soc. 134 (2005), 11531160 .

[25] M. E. H. Ismail, Classical and Quantum Orthogonal Polynomials in one Variable, Cambridge University Press, paperback edition, Cambridge, 2009.

[26] E. J. Heller and W. P. Reinhardt and H. A. Yamani, On an equivalent quadrature calculation of matrix elements of $\left(z-P^{2} / 2 m\right)$ using an $L^{2}$ expansion technique, $\mathrm{j}$ J. Comp. Phys. 13 (1973), 536-549.

[27] M. E. H. Ismail and X. Li, Bounds for extreme zeros of orthogonal polynomials, Proc. Amer. Math. Soc. 115 (1992) , 131-140.

[28] M. E. H. Ismail and D. R. Masson, q-Hermite polynomials, biorthogonal rational functions, and q-beta integrals, Transactions Amer. Math. Soc. 346 (1994), 63116.

[29] M. E. H. Ismail and M. E. Muldoon, Inequalities and monotonicity properties for gamma and q-gamma functions, in "Approximation and Computation: A Festschrift in Honor of Walter Gautschi”, R. V. M. Zahar, ed., Birkhäuser, BostonBasel-Berlin, 1994, pp. 309-323.

[30] J.R. Klauder and E.C.G. Sudarshan, Fundamentals of Quantum Optics, Benjamin, New York, 1968.

[31] R. Koekoek and R. Swarttouw, The Askey-scheme of hypergeometric orthogonal polynomials and its q-analogues, Reports of the Faculty of Technical Mathematics and Informatics no. 98-17, Delft University of Technology, Delft, 1998.

[32] C.T. Munger, Ideal basis sets for the Dirac Coulomb problem: eigenvalue bounds and convergence proofs, J. Math. Phys. 48 (2007) 022301 
[33] P. Nevai, Orthogonal polynomials, Memoirs Amer. Math. Soc. 18 Number 213, 1979, 185 pages.

[34] A. Odzijewicz, Coherent states and geometric quantization, Commun. Math. Phys. 150 (1992), 38 - 413.

[35] A. Odzijewicz, M. Horowski and A. Tereszkiewicz, Integrable multi-boson systems and orthogonal polynomials, J. Phys. A: Math. Gen. 34 (2001), 4353-4376.

[36] E. D. Rainville, Special Functions, Macmillan, New York, 1960.

[37] E. Schrodinger, Der stetige Ubergang von der Mikro- zur Makromechanik, Naturwiss. 14 (1926), 664 - 666.

[38] G. Szegö, Orthogonal Polynomials, Fourth Edition, Amer. Math. Soc., Providence, 1975.

[39] S. Sivakumar, Studies on nonlinear coherent states, J. Opt. B: Quantum Semiclass. Opt. 2 (2000), R61R75.

[40] D. V. Widder, The Laplace Transform, Princeton University Press, Princeton, NJ, 1941.

[41] H.A. Yamani, W.P. Reinhardt, $L^{2}$ discretizations of the continuum: radial kinetic energy, Coulomb Hamiltonian, Phys. Rev. A 11 (1975) 1144-1156.

Email: M.E.H.I. : mourad.eh.ismail@gmail.com, S.T.A. : stali@mathstat.concordia.ca 